\title{
Illness episodes in a cohort of preterm infants in their first year of life
}

\author{
S J Muller, ${ }^{1}$ BSc; H J Zar, ${ }^{1}$ FRCPaeds, PhD; L Tooke, ${ }^{2}$ FCPaeds, Cert (Neonatol) \\ ${ }^{1}$ Department of Paediatrics and Child Health, University of Cape Town, South Africa \\ ${ }^{2}$ Groote Schuur Neonatal Unit, Department of Paediatrics and Child Health, University of Cape Town, South Africa
}

Corresponding author: S J Muller (mullerseth@gmail.com)

\begin{abstract}
Background. There are limited data available regarding the illness episodes and hospital admissions of preterm infants after initial discharge in low- and middle-income countries.

Objective. To prospectively follow a cohort of HIV-unexposed preterm infants (29 - 34 weeks) and describe their illness episodes, admissions and associated risk factors over a one-year period.

Methods. The study was nested in a parent study evaluating the efficacy of a monoclonal antibody against respiratory syncytial virus from January 2017 to March 2017, comprising 53 infants enrolled from two government neonatal nurseries in Cape Town, South Africa. Descriptive data were collected regarding perinatal history and socioeconomic factors of the infants' households. All infants received careful follow-up. Logistic regression was performed to assess the association between hospitalisation and socioeconomic factors.

Results. All 53 infants who were recruited were followed up over one year. There were 436 illness episodes, of which 31 were hospital admissions. One infant died at home. The most common illnesses were respiratory (53\%) and dermatological (17\%) in nature. Lower respiratory tract infections (LRTIs) accounted for $71 \%$ of all hospital admissions. There were no significant associations between socioeconomic subgroups when comparing illness episodes or hospital admissions.

Conclusion. This study is one of the few to record all illness episodes and not solely admissions over a one-year period for HIV-unexposed infants. There are high rates of intercurrent respiratory infection and hospitalisation of preterm infants in their first year of life. Public health interventions to reduce the risk of LRTI must be strengthened. Larger studies need to be done to be able to report on the associations with socioeconomic determinants in developing countries.
\end{abstract}

S Afr J Child Health 2021;15(1):44-49. https://doi.org/10.7196/SAJCH.2021.v15i1.1782

Preterm infants account for a large and increasing proportion of neonatal and childhood morbidity and mortality worldwide, especially in low- and middle-income countries (LMICs). ${ }^{[1]}$ However, limited data are available regarding the outcomes of preterm infants after discharge in these countries. ${ }^{[2,3]}$

The aim of the present study was to provide a descriptive analysis of the outcomes of a cohort of preterm infants from two public neonatal intensive care units (NICUs) in South Africa (SA) through one year of follow-up.

\section{Methods}

The study was nested in a prospective cohort study of preterm infants born between 29 and 35 weeks' gestational age at Groote Schuur Hospital (GSH) and Mowbray Maternity Hospital (MMH) in Cape Town, who were enrolled in a multicentre phase $2 \mathrm{~b}$ novel respiratory syncytical virus (RSV) monoclonal antibody trial. This double-blinded randomised controlled trial (RCT) used a ratio of intervention to placebo of 2:1. Gestational age was determined by an ultrasound at less than 20 weeks' gestation, or foot length at birth. ${ }^{[4]}$

Infants were enrolled following delivery at GSH or MMH neonatal units from 1 January 2017 to 31 March 2017. Inclusion criteria were healthy infants born between 29 weeks 0 days' and 34 weeks 6 days' gestational age and maternal informed consent. Exclusion criteria for the parent study were:

- if caregivers were unable to follow up or if there was doubt regarding follow-ups, e.g. extreme poverty, adoption, significant medical or mental illness, opioid drug addiction or maternal age $<18$ years

- maternal HIV infection
- any acute illnesses, blood products or drug therapy two weeks prior to enrolment (except multivitamins, iron or probiotics)

- any known chronic illness, except for children with uncomplicated congenital heart disease (e.g. patent ductus arteriosus, small septal defect).

All participants from the parent study were eligible for the nested study. Follow-up was at the research centre at Red Cross War Memorial Children's Hospital (RXH) on days 8, 31, 91, 151 and 361 after discharge. Clinical and socioeconomic data, feeding choice and any illness, clinic visits or hospital admission were obtained at each follow-up visit. Exclusive breast milk was defined as no other food or drink, not even water. The infants' folders were also reviewed to obtain perinatal data. Data were collected via a standardised questionnaire completed by the study staff at each follow-up visit.

To ensure no illnesses were missed, the following measures in addition to routine visits were put into place:

- in case of illness, emergencies or any adverse incident, caregivers made telephonic contact (available 24 hours a day) with the research team

- such calls were logged, advice was given, and infants were followed up as either in- or outpatients, where further information was collected

- caregivers were phoned monthly to inquire about any symptoms or illnesses.

The primary outcome for the RSV monoclonal study was medically attended RSV-lower respiratory tract infection (LRTI). 
For this nested study, the primary outcome was to describe maternal and infant characteristics and all infant intercurrent illness episodes through one year after discharge.

Ethical approval for the nested study was granted by the Human Research Ethics Committee, Faculty of Health Sciences, University of Cape Town (ref. no. 886/2016). All mothers provided written consent prior to being included in the study.

\section{Statistical analyses}

Data were analysed using RStudio version 1.1.463 (RStudio, USA). Continuous variables were summarised as medians with interquartile range (IQR) for non-parametric data. Categorical variables were expressed as frequencies and percentages. To assess whether there were associations between socioeconomic factors and hospital admissions, a logistic linear regression was done. Gestational age and birthweight were adjusted for in the logistic regression. A $p$-value $<0.05$ was interpreted as statistically significant.

\section{Results}

\section{Enrolment and follow-up}

Fifty-six infants were enrolled in the RSV monoclonal study from Groote Schuur and Mowbray Maternity Hospitals; all were offered participation in the follow-up study, of whom three declined. The study therefore included 53 preterm infants, of whom there were four sets of twins. No infant was lost to follow-up during the one-year period, but one infant died at 130 days of age (12 weeks corrected age) at home from presumed pneumonia.

\section{Maternal characteristics}

The median age of the mothers at time of delivery was 28 years, with five mothers being less than 20 years of age. Table 1 describes the maternal characteristics. The majority of mothers $(n=35 ; 71 \%)$ were living in formal housing and all had access to electricity; 43 (88\%) had some high school education and in four $(8 \%)$ the highest level of education was primary school. The median (IQR) number of people living in the same dwelling, including infants, was 7 (5 - 8). Table 1 presents maternal medical and socioeconomic backgrounds. A third of mothers reported household smoke exposure and $14 \%$ reported substance abuse during pregnancy.

\section{Infant characteristics}

Most infants $(n=35 ; 66 \%)$ were born by emergency caesarean section, with a median birthweight of $1500 \mathrm{~g}$ (Table 2). The medin lengtha of stay after birth was 27 days.

Two infants had congenital abnormalities: one had upper limb dysgenesis and another had a ventricular septal defect (VSD). Three infants were diagnosed with patent ductus arteriosis (PDA) via echocardiogram, all of which spontaneously closed.

The number of infants who had fathers who provided financially was $43(81 \%)$ at day 8 and gradually declined to $37(70 \%)$ by day 361 . Fathers lived in the same dwelling at birth for 31 (58\%) of the infants, with a gradual decline to 27 (51\%). Substance misuse (opioids, marijuana and amphetamines) fluctuated between $2 \%$ and $9 \%$ of the households. At one year, only four infants were attending crèche; all the others were cared for by family members.

A high proportion of infants received exclusively breast milk (EBM) from birth until discharge $(n=50 ; 94 \%)$ with a gradual reduction over time (Fig. 1). By day 151, 9 infants (17\%) received EBM and 19 infants (37\%) received any breast milk. By day 361, there were still 19 infants (37\%) receiving any breast milk.

The median (IQR) weight for corrected age Z-scores were -0.58 $(-1.52-0.08)$ on day $31,-0.21(-0.85-0.29)$ on day 91 and -0.49
(-1.08 - 0.07) on day 151. Another infant was admitted with severe acute malnutrition at 10 months of age.

By day $361,91 \%$ of infants had received all their vaccines including the 9-months measles and pneumococcal vaccines.

\section{Intercurrent illness episodes and hospital admissions}

The total number of illness episodes reported over the study period was 436, with a median (IQR) of $7(5-11)$ illness episodes per infant per year (e/iy) (Table 3$)$. The most common illness $(n=170$; $39 \%$ ) was upper respiratory tract infection (URTI), with a rate of 3.2 e/iy. Seventy-two (17\%) illness episodes were attributed to dermatologicalconditions, with a rate of 1.4 e/iy and $59(14 \%)$ illness

\section{Table 1. Characteristics of mothers}

\begin{tabular}{|c|c|}
\hline Outcome & $N=49, n(\%)^{*}$ \\
\hline Maternal age at time of delivery, median (IQR) & $28(23-32)$ \\
\hline Attended antenatal care & $43(88)$ \\
\hline \multicolumn{2}{|l|}{ Booking bloods } \\
\hline Positive rapid plasma reagin & $1(2)$ \\
\hline Rhesus negative & $3(6)$ \\
\hline Gravidity, median (IQR) & $1(1-2)$ \\
\hline \multicolumn{2}{|l|}{ Antenatal treatment } \\
\hline None & $20(41)$ \\
\hline Steroids & $18(37)$ \\
\hline Magnesium sulphate & $6(12)$ \\
\hline Steroids and magnesium sulphate & $5(10)$ \\
\hline \multicolumn{2}{|l|}{ Maternal illnesses during pregnancy } \\
\hline Pre-eclampsia/eclampsia & $8(16)$ \\
\hline Sepsis/infection & $7(14)$ \\
\hline \multicolumn{2}{|l|}{ Maternal illnesses known prior to pregnancy } \\
\hline Cardiac & $2(4)$ \\
\hline Lung disease & $3(6)$ \\
\hline Endocrine & $2(4)$ \\
\hline Other & $4(8)$ \\
\hline \multicolumn{2}{|l|}{ Housing } \\
\hline Shack & $10(21)$ \\
\hline Wendy house & $4(8)$ \\
\hline Apartment & $8(16)$ \\
\hline Free-standing house & $27(55)$ \\
\hline \multicolumn{2}{|l|}{ Access to water } \\
\hline Piped inside the house & $36(73)$ \\
\hline Piped outside the house & $9(18)$ \\
\hline Communal tap outside the yard & $4(9)$ \\
\hline \multicolumn{2}{|l|}{ Level of education of mother } \\
\hline Grade $0-7$ & $4(8)$ \\
\hline Grade 8 - 12 & $40(82)$ \\
\hline Diploma/degree & $5(10)$ \\
\hline \multicolumn{2}{|l|}{ Yearly income of household, ZAR } \\
\hline$<99999$ & $44(90)$ \\
\hline $100000-350000$ & $1(2)$ \\
\hline$>350001$ & $4(8)$ \\
\hline \multicolumn{2}{|l|}{ Smoking in the home } \\
\hline No & $33(67)$ \\
\hline Yes & $16(33)$ \\
\hline Substance abuse in the home ${ }^{\dagger}$ & $7(14)$ \\
\hline $\begin{array}{l}\mathrm{IQR}=\text { interquartile range. } \\
{ }^{*} \text { Unless otherwise specified. } \\
{ }^{\circ} \text { Opioids, marijuana and amphetamines. }\end{array}$ & \\
\hline
\end{tabular}




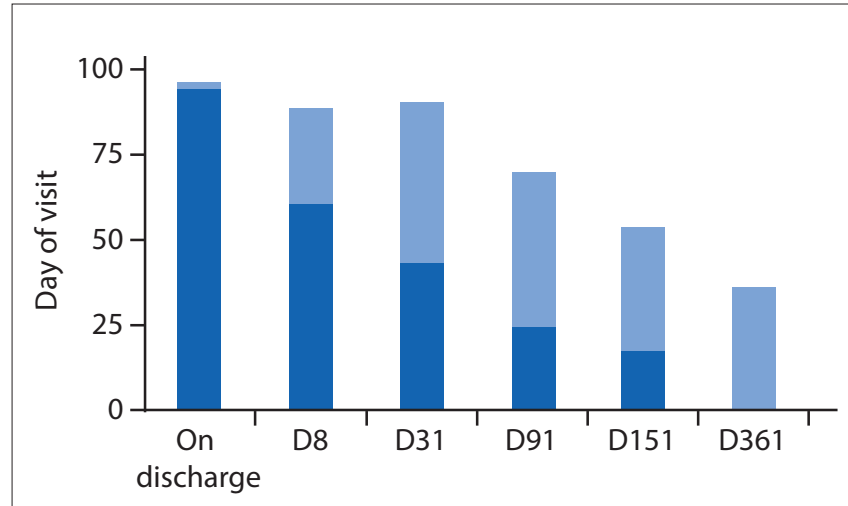

Infants, \%

Any breast milk Exclusive breast milk

Fig. 1. Percentage of infants who received exclusive and any breast milk over one year.

Table 2. Infant characteristics

\begin{tabular}{|c|c|}
\hline Outcome & $N=53, n(\%)^{*}$ \\
\hline \multicolumn{2}{|l|}{ Mode of delivery } \\
\hline Caesarean section & $35(66)$ \\
\hline Vaginal delivery & $18(34)$ \\
\hline LOS after birth (days), median (IQR) & $27(23-31)$ \\
\hline Male & $32(60)$ \\
\hline Birthweight, g (median (IQR)) & $1500(1300-1840)$ \\
\hline GA at birth, weeks ${ }^{+d a y s}$ (median (IQR)) & 32 weeks $\left(30^{+3}-33^{+2}\right)$ \\
\hline $\begin{array}{l}\text { Head circumference for age at birth, Z-score } \\
(\text { median }(\mathrm{IQR}))\end{array}$ & $0.04(-1.07-0.61)$ \\
\hline \multicolumn{2}{|l|}{ Apgar scores, median (IQR) } \\
\hline 1 minute & $7(5-8)$ \\
\hline 5 minutes & $9(8-10)$ \\
\hline \multicolumn{2}{|l|}{ Initial resuscitation required } \\
\hline None & $27(51)$ \\
\hline Oxygen & $1(2)$ \\
\hline Facemask ventilation & $17(32)$ \\
\hline Cardiopulmonary resuscitation & $5(9)$ \\
\hline Intubation & $2(4)$ \\
\hline Epinephrine & $1(2)$ \\
\hline Surfactant required & $2(4)$ \\
\hline Temperature at admission, median (IQR) & $36.1(35.9-36.4)$ \\
\hline Congenital abnormalities & $2(4)$ \\
\hline \multicolumn{2}{|l|}{ Highest level of respiratory support } \\
\hline Room air & $18(34)$ \\
\hline Nasal prong oxygen & $3(6)$ \\
\hline High-flow nasal cannula & $7(13)$ \\
\hline Nasal CPAP & $22(42)$ \\
\hline Mechanical ventilation & $3(6)$ \\
\hline \multicolumn{2}{|l|}{ Feeding at discharge } \\
\hline Exclusive breast milk & $50(94)$ \\
\hline Breast milk substitute & $2(4)$ \\
\hline Mixed & $1(2)$ \\
\hline Twins & $4(8)$ \\
\hline
\end{tabular}

episodes with a rate of 1.1 e/iy for both lower respiratory tract infection (LRTI) and gastrointestinal disorders.

Over the one year of follow-up, 20 (38\%) infants were admitted to hospital, with a total of 31 admissions. Of the 31 admissions, 29 were managed medically and two required surgical interventions (repair of a VSD and drainage of an abscess). Four required admission to ICU. LRTI accounted for 22 (71\%) of all hospital admissions (Fig. 2), with a rate of 0.4 e/iy. The median (IQR) corrected age of hospital admissions was 12 weeks $^{+3}$ days $\left(5^{+2}-28^{+2}\right)$ and the median (IQR) length of hospital stay was 5 days $(3-7)$. There was no association between corrected gestational age and timing of hospital admissions.

One infant with presumed pneumonia died at home, giving an overall mortality rate of 1 in 53 (2\%); no postmortem examination was permitted.

\section{Factors associated with hospitalisation}

Table 4 shows the multivariate logistic regression of factors associated with hospital admission. One infant with a VSD had a large number of illness episodes and hospital admissions unrelated to prematurity, so this infant was excluded from the multivariate analysis.

\section{Discussion}

This study has shown a high prevalence of poor socioeconomic factors and smoke exposure as well as a high number of hospitalisations in this group of SA preterm infants, but low mortality. There were no associations between hospital admissions and socioeconomic factors, but this may partly be due to the relatively poor socioeconomic status of all participants, with no participants from high socioeconomic groups. Further, the small sample size limits the power of the analyses to detect such associations.

Due to the rigorous follow-up, this is one of the few studies to accurately record all illness episodes and not solely admissions to hospital. Respiratory tract infections (URTI and LRTI) contributed just over half of all illness episodes. Of the 229 respiratory tract infections recorded, only 23 (10\%) required admission to hospital.

More than a third of infants were admitted to hospital over the one-year follow-up. These results are higher than those from a systematic review ${ }^{[6]}$ in which $25 \%$ of low- or very low-birthweight infants required hospitalisation in the first year of life; however, the studies included in the systematic review were done in high-income countries. The reason for the higher rate of hospital admissions in this population could be due to a higher burden of infectious

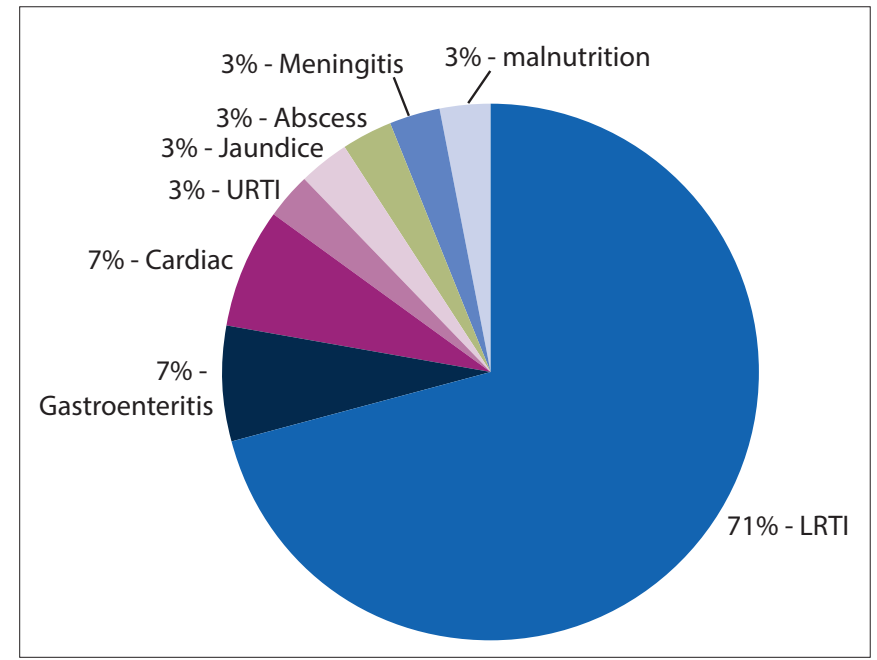

Fig. 2. Reasons for hospital admissions. (URTI = upper respiratory tract infection; $L R T I=$ lower respiratory tract infection) . 
Table 3. Illness episodes in infants through one year of age

\begin{tabular}{|c|c|}
\hline Type of illness & $\begin{array}{l}\text { Illness episodes, } \\
N=436, n^{*}\end{array}$ \\
\hline Respiratory, $n(\%)$ & $229(53)$ \\
\hline URTI & 170 \\
\hline LRTI & 59 \\
\hline Dermatological, $n(\%)$ & $72(17)$ \\
\hline Napkin dermatitis & 12 \\
\hline Viral exanthem & 12 \\
\hline Other & 11 \\
\hline Falliculitis/impetigo & 9 \\
\hline Fungal & 7 \\
\hline Scabies & 7 \\
\hline Papular urticuria & 7 \\
\hline Tinea & 4 \\
\hline Mouth ulcers & 3 \\
\hline Gastrointestinal, $n(\%)$ & $59(14)$ \\
\hline Gastroenteritis & 43 \\
\hline Reflux & 5 \\
\hline Oral thrush & 5 \\
\hline Constipation & 2 \\
\hline Dysentery & 2 \\
\hline Abdominal cramps & 2 \\
\hline Ears, nose and throat, $n(\%)$ & $15(3)$ \\
\hline Otitis media & 13 \\
\hline Tonsillitis & 1 \\
\hline Laryngomalacia & 1 \\
\hline Haematological, $n$ (\%) & $12(3)$ \\
\hline Anaemia & 9 \\
\hline Jaundice & 3 \\
\hline Ophthalmological, $n(\%)$ & $10(2)$ \\
\hline Conjunctivitis & 7 \\
\hline Blocked duct & 3 \\
\hline Other, $n(\%)$ & $10(2)$ \\
\hline Fever & 5 \\
\hline Colic & 3 \\
\hline Lymphadenopathy & 2 \\
\hline Surgical, $n(\%)$ & $9(2)$ \\
\hline Burn & 2 \\
\hline Umbilical hernia & 2 \\
\hline Abscess & 2 \\
\hline Undescended testes & 1 \\
\hline Surgical complications & 1 \\
\hline Cardiac, $n(\%)$ & $6(1)$ \\
\hline Allergy, $n(\%)$ & $5(1)$ \\
\hline Eczema & 3 \\
\hline Allergic rhinitis & 2 \\
\hline Central nervous system, $n(\%)$ & $3(1)$ \\
\hline Meningitis & 1 \\
\hline Torticollis & 1 \\
\hline Macrocephaly & 1 \\
\hline Urology, $n(\%)$ & $3(1)$ \\
\hline Malnutrition ${ }^{+}$ & $2(1)$ \\
\hline \multicolumn{2}{|c|}{$\begin{array}{l}\text { URTI }=\text { upper respiratory tract infection; LRTI }=\text { lower respiratory tract infection. } \\
{ }^{*} \text { Unless otherwise specified. } \\
\text { 'Defined by WHO as severe and moderate acute malnutrition. }{ }^{5}\end{array}$} \\
\hline
\end{tabular}

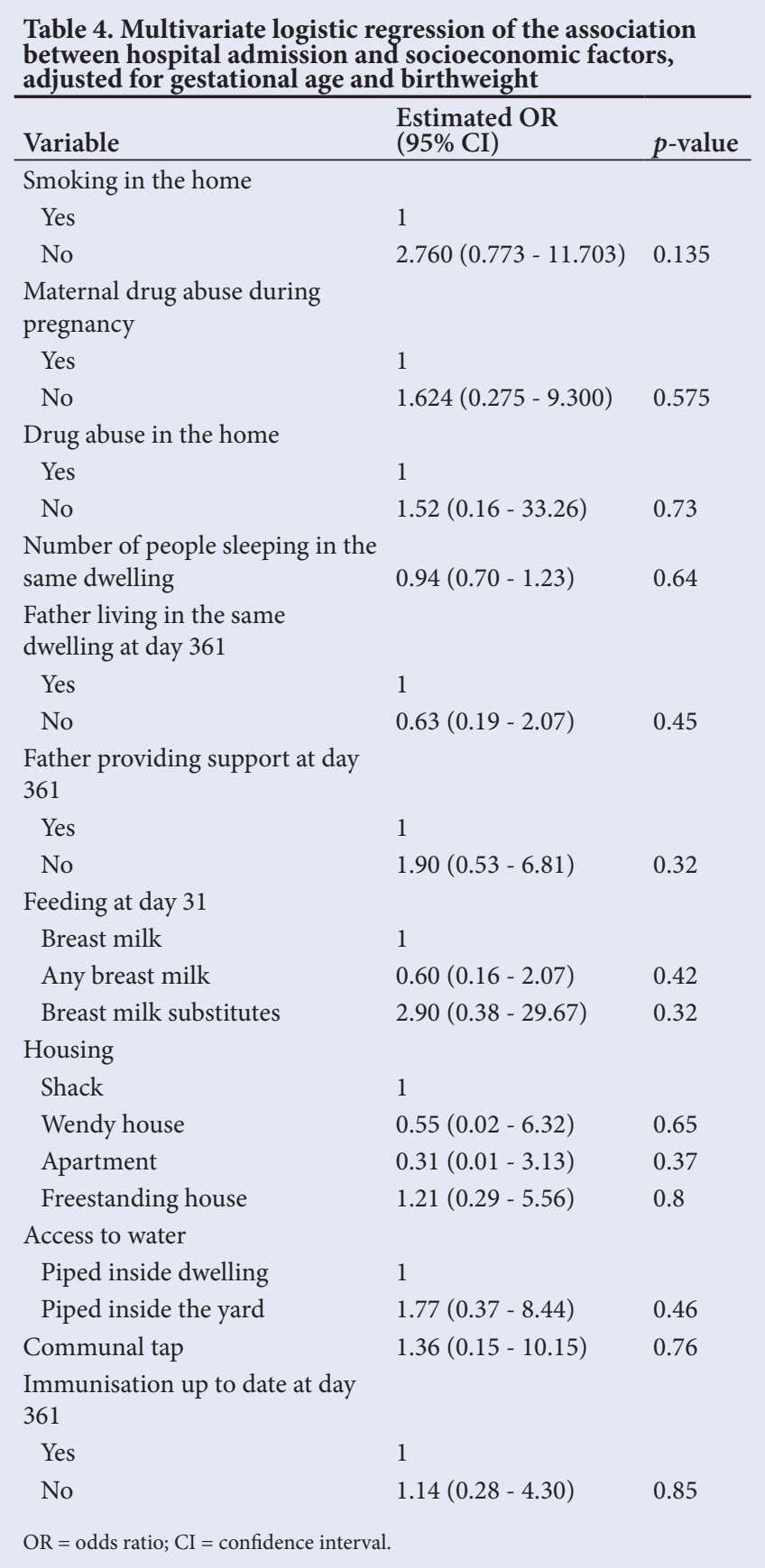

diseases in LMICs and higher exposure to risk factors; this is especially true for severe LRTI as a major cause of hospitalisation, which is consistent with studies from other settings. ${ }^{[7,8]}$ The high burden of respiratory conditions highlights the need to strengthen preventive interventions and new strategies to tackle this burden on the health sector.

Environmental exposures that may be associated with LRTI include smoke exposure. ${ }^{[9-11]}$ Of concern is that a third of infants (33\%) in the present study were exposed to household smoke. Crowding may be another factor; the household size in this study was higher than the 4.9 mean household size for people living with children in South Africa in 2018. ${ }^{[12]}$ Lack of breastfeeding may be an additional risk factor. Despite the WHO recommendations for EBM until six months of age, this study again demonstrates the low rates of EBM, in SA. By day 151, only 9 (17\%) of the infants received EBM 
which is similar to another local study where only $12 \%$ received EBM. ${ }^{[13,14]}$ Encouragingly, most of the infants were transitioned to solid feeds after 6 months. In a Lancet systematic review from 2016, it was found that breastfeeding reduced diarrhoea episodes by $~ 50 \%$ and further reduced hospital admissions for diarrhoea by $72 \%$. Breastfeeding also reduced $33 \%$ of respiratory infections and $57 \%$ of hospital admissions owing to respiratory infections. ${ }^{[15]}$ Even though exclusive breastfeeding is the ideal, any breastfeeding should be encouraged as there is a modest protective dose response effect with partial breastfeeding when compared with non-breastfeeding. ${ }^{[16]}$

Lack of immunisations makes a child more vulnerable to LRTI, and preterm infants should be vaccinated according to their chronological and not corrected age. ${ }^{[17]}$ Only $9 \%$ of infants in this study were not up to date with their vaccines, which is lower than the average of $19 \%$ of infants with incomplete vaccination reported in Western Cape Province. ${ }^{[14]}$

Poor levels of parental education have been shown to be associated with worse outcomes and an increased risk for preterm infant mortality. ${ }^{[18]}$ Only 6 of the infants' mothers in the present study had a form of post-school qualification. There was, however, a low mortality rate of $2 \%$ in this study. In contrast, a study in 1997 , following very-low-birthweight (VLBW) infants in SA over 18 months, reported a $13 \%$ mortality rate. ${ }^{[19]}$ The lower mortality rate in the present study may reflect improvements in care owing to advances in technology and maternal and infant management. Further, the high coverage of childhood immunisations, exclusion of HIV-infected mothers and regular follow-up through the study may all have contributed to improved outcomes.

No association was shown for infant morbidity in those families where substance abuse was reported; however, there may have been under-reporting owing to the fluctuating reported numbers and the prevalence being well below the national average of $13 \% .{ }^{[20]}$

It is surprising that in such a closely monitored group, two infants developed severe malnutrition, but this highlights the constant threat of food security in many of our communities.

\section{Study strengths and limitations}

A strength of the study is the comprehensive follow-up and the ability to identify all intercurrent illness episodes and not only hospital admissions over a year for each infant.

This study was nested in a much larger funded study and, while this resulted in excellent follow-up, there are three important limitations which hinder the generalisation of the results.

The first limitation is that all babies were HIV unexposed (HU). It has been shown that HIV-exposed uninfected (HEU) infants have an increased infectious morbidity when compared with HU infants. ${ }^{[21]}$ There has been debate that many of the earlier studies were done before the move to early universal maternal antiretroviral therapy (ART) and exclusive breastfeeding, which may ameliorate some of the mechanisms responsible for these earlier findings. Le Roux et al. ${ }^{[22]}$ showed that even with maternal ART and universal breastfeeding, HEU infants have an increased incidence ratio of infection-related hospitalisation when compared with HU infants, but this was significant only in the first three months of life.

Although the efficacy of the monoclonal antibody in the parent study is still unknown (results have not yet been published), it is possible that those infants who received the antibody would have been less likely to consult or be admitted for complications of RSV. Other RSV monoclonal antibodies such as palivizumab and motavizumab have been shown to decrease hospital admissions for RSV by $50 \%$ as well as decrease outpatient visits for LRTI. ${ }^{[23,24]}$
Finally, the 'most at risk infants' could have been excluded owing to inclusion criteria ruling out mothers who were unlikely to come to follow-up visits. Linked to this factor is that because participants were part of a study follow-up and had access to care, outcomes could differ from those patients without such access.

Despite these limitations, this study remains important as it shows not only hospital admissions but also the high number of illness episodes in this cohort of preterm infants. It is probable that these results are an under-representation of what could be anticipated in the general population, especially if higher-risk infants are included and monoclonal antibodies are not available.

\section{Conclusion}

There are high rates of intercurrent infection and hospitalisation in $\mathrm{HU}$ preterm infants in their first year of life. Public health interventions to reduce the risk of LRTI must be strengthened, including promoting exclusive breastfeeding, avoidance of tobacco smoke exposure, and improvements in living conditions. Larger studies are needed to investigate morbidity and socioeconomic determinants.

\section{Declaration. None.}

Acknowledgements. The authors thank the staff of Research Centre for Adolescents and Child Health (REACH) as well as the mothers and their infants who participated.

Author contributions. SM, HZ and LT designed the study. SM collected and analysed the data. SM drafted the manuscript and it was critically revised by LT and HZ.

Funding. The parent study received research funding from MedImmune, LLC, a wholly owned subsidiary of AstraZeneca PLC. HZ is supported by the SA MRC.

Conflicts of interest. None.

1. Grisaru-Granovsky S, Reichman B, Lerner-Geva L, Boyko V. Mortality and morbidity in preterm small-for-gestational-age infants: A population-based study. Am J Obstet Gynocology 2012;206(2):150.e1-150.e7.

2. Cooper PA, Rothberg AD, Thomson PD, Cohn RJ. The spectrum of clinical problems and outcome in infants in a neonatal unit. S Afr Med J 1983;64(15):574-577.

3. Campbell A, Rudan I. Systematic review of birth cohort studies in Africa. J Glob Health 2011;1(1):46-58.

4. Drey EA, Kang M, Mcfarland W, Darney PD. Improving the accuracy of fetal foot length to confirm gestational duration. Obstet Gynecol 2005;105(4):773778. https://doi.org/10.1097/01.aog.0000154159.75022.11

5. World Health Organization, United Nations Children's Fund. WHO child growth standards and the identification of severe acute malnutrition in infants and children. New York: United Nations Children's Fund; 2009.

6. Petrou S, Sach T, Davidson L. The long-term costs of preterm birth and low birth weight: Results of a systematic review. Child Care Heal Dev 2000;27(2):97-115. https://doi.org/10.1046/j.1365-2214.2001.00203.x

7. LeRoux DM, Nicol MP, Myer L, et al. Lower respiratory tract infections in children in a well-vaccinated South African birth cohort: Spectrum of disease and risk factors. CID 2019;69(9):1588-1596. https://doi.org/10.1093/cid/ciz017

8. Martens P, Dersen S, Gupta S. Predictors of hospital readmission of Manitoba newborns within six weeks postbirth discharge - a population-based study Pediatrics 2004;114(3):708-713. https://doi.org/10.1542/peds.2003-0714-1

9. Vanker A, Barnett W, Workman L, et al. Early-life exposure to indoor air pollution or tobacco smoke and lower respiratory tract illness and wheezing in African infants: A longitudinal birth cohort study. Lancet Planet Heal 2017;1(8):e328-336. https://doi.org/10.1016/s2542-5196(17)30134-1

10. Li JSM, Peat JK, Xuan W, Berry G. Meta-analysis on the association between environmental tobacco smoke (ETS) exposure and the prevalence of lower respiratory tract infection in early childhood. Pediatr Pulmono 1999;27:5-13. https://doi.org/10.1002/(sici)1099-0496(199901)27:1\%3C5::aidppul3\%3E3.0.co;2-5

11. Reddy P, Zuma K, Shisana O, et al. Prevalence of tobacco use among adults in South Africa: Results from the first South African National Health and Nutrition Examination Survey. S Afr Med J 2015;105(8):648-655. https://doi. org/10.7196/samjnew.7932 
12. Hall K. Children's access to housing. In: Shung-king M, Lake L, Saunders D, Hendricks M, editors. South African Child Gauge 2019. Cape Town: Children's Institute, University of Cape Town; 2019. p. 248-251.

13. Siziba LP, Jerling J. Low rates of exclusive breastfeeding are still evident in four South African provinces. South Afr J Clin Nutr 2015;28(4):170-179.

14. Shung-king M, Lake L, Sanders D, Hendricks M. Child and adolescent health. South African Child Gauge 2019. Cape Town: Children's Institute, University of Cape Town; 2019.

15. Victora CG, Bahl R, Barros AJD, et al. Breastfeeding in the 21st century: Epidemiology, mechanisms, and lifelong effect. Lancet 2016;387:475-490. https://doi.org/10.1016/s0140-6736(15)01024-7

16. Sankar MJ, Sinha B, Chowdhury R, et al. Optimal breastfeeding practices and infant and child mortality: A systematic review and meta-analysis. Acta Paediatr 2015;104:3-13. https://doi.org/10.1111/apa.13147

17. Tooke L, Louw B. A successful preterm vaccination program in a neonatal unit in a developing country. Heliyon 2019;5(11):1-3. https://doi.org/10.1016/j. heliyon.2019.e02857

18. Ko Y, Shin S, Park SM, et al. Effects of employment and education on preterm and full-term infant mortality in Korea. Public Health 2014;128:254-261. https://doi.org/10.1016/j.puhe.2013.12.010

19. Cooper PA, Sandler DL. Outcome of very low birth weight infants at 12 to 18 months of age in Soweto, South Africa. Pediatrics 1997;99(4):537-544. https:// doi.org/10.1542/peds.99.4.537
20. Myers B, Petersen P, Govender R, Manderscheid R, Koch JR. Substance abuse treatment engagement, completion and short-term outcomes in the Western Cape Province, South Africa: Findings from the Service Quality Measures Initiative. Drug Alcohol Depend 2019;185:278-284. https://doi.org/10.1016/j. drugalcdep.2017.12.033

21. Slogrove AL, Goetghebuer T, Cotton MF, Singer J, Bettinger JA. Pattern of infectious morbidity in HIV-exposed uninfected infants and children. Front Immunol 2016;7:164. https://doi.org/10.3389/fimmu.2016.00164

22. Le Roux SM, Abrams EJ, Donald KA, et al. Infectious morbidity of breastfed, HIV-exposed uninfected infants under conditions of universal antiretroviral therapy in South Africa: A prospective cohort study. Lancet Child Adolesc Heal 2020;4(3):220-231. https://doi.org/10.1016/S2352-4642(19)30375-X

23. The IMpact-RSV Study Group. Palivizumab, a humanised respiratory syncytial virus monoclonal antibody, reduces hospitalisation from respiratory syncytial virus infection in high-risk infants. Pediatrics 1998;102(3):531-537.

24. Carbonell-Estrany AX, Eric AF. Motavizumab for prophylaxis of respiratory syncytial virus in high-risk children: A noninferiority trial. Pediatrics 2010;125(1):e35-e51. https://doi.org/10.1542/peds.2008-1036

Accepted 3 September 2020. 